\title{
AMBIGUITY IN THE DETERMINATION OF THE FREE ENERGY ASSOCIATED WITH THE CRITICAL CIRCLE MAP
}

\author{
BRIAN G. KENNY ${ }^{\bowtie 1}$ and TONY W. DIXON ${ }^{2}$
}

(Received 5 July, 2006; revised 19 October, 2006)

\begin{abstract}
We consider a simple model to describe the widths of the mode-locked intervals for the critical circle map. By using two different partitions of the rational numbers based on Farey series and Farey tree levels, respectively, we calculate the free energy analytically at selected points for each partition. It emerges that the result of the calculation depends on the method of partition. An implication of this finding is that the generalized dimensions $D_{q}$ are different for the two types of partition except when $q=0$; that is, only the Hausdorff dimension is the same in both cases.
\end{abstract}

2000 Mathematics subject classification: primary 37-XX; secondary 37E10, 37D35, $37 \mathrm{~L} 30$.

Keywords and phrases: circle maps, thermodynamic formalism, Farey trees, Farey series.

On the basis of their numerical investigations, Jensen et al. $[9,10]$ conjectured that for the critical circle map, the set of irrational windings is a multifractal set characterized by a universal Hausdorff dimension $D_{H}$. In order to better characterize such sets, different thermodynamic formalisms [4, 6] have been introduced and, with them, the idea of generalized dimensions [8]. In this communication we point out that for the circle map, such a description is not unique and depends upon the details of how the set is partitioned. However, the Hausdorff dimension is the same for two distinct partitions.

To illustrate this point, we shall examine a simple model for the widths of the modelocked intervals of the circle map, and show that two different partitions of the rationals lead to two different functional forms for the free energy $F(\beta)$ and hence to different

\footnotetext{
${ }^{1}$ Department of Theoretical Physics, Research School of Physical Science and Engineering, Australian National University, Canberra, ACT 0200, Australia; e-mail: brianikenny@anu.edu.au. ${ }^{2}$ Formerly at School of Mathematics \& Statistics, Curtin University of Technology, GPO Box U1987, Perth,WA 6845, Australia; e-mail: tony.w.dixon@gmail.com.

(C) Australian Mathematical Society 2009, Serial-fee code 1446-1811/2009 \$16.00
} 
values of $D_{q}$. Suppose that the width $\Delta(P / Q)$ of the mode-locked interval for the critical circle map associated with the winding number $P / Q$ is given by

$$
\Delta\left(\frac{P}{Q}\right)=k Q^{-\delta}
$$

independent of $P$ (see [11]; this model for the width of the mode locked regions is a simpler version of a suggestion made by Lanford). Here $P$ and $Q$ are coprime integers, and $k, \delta$ are constants (we assume that $\delta>2$ ).

In this model, it is possible to analytically calculate the total sum of the widths of the mode-locked intervals (which is taken to be one at criticality):

$$
\sum_{Q} \sum_{P} \Delta\left(\frac{P}{Q}\right)=k \sum_{Q=1}^{\infty} \phi(Q) Q^{-\delta}=k \frac{\zeta(\delta-1)}{\zeta(\delta)}=1,
$$

where $\zeta$ is the Riemann zeta function and $\phi(Q)$ is the Euler function [7]. The sum in (1.1) is taken over all irreducible rationals $P / Q$ with $P<Q$, and $\Delta(P / Q)$ is the width of the parameter interval for which the iterates of a critical circle map lock on to a cycle of length $Q$, with winding number $P / Q$. Therefore, $k$ is fixed to be

$$
k=\frac{\zeta(\delta)}{\zeta(\delta-1)}
$$

Now, the Hausdorff dimension is the largest value of $\beta$ for which the sum

$$
Z(\beta)=\sum_{Q} \sum_{P}\left[\Delta\left(\frac{P}{Q}\right)\right]^{\beta}=k^{\beta} \sum_{Q} \phi(Q) Q^{-\delta \beta}=k^{\beta} \frac{\zeta(\delta \beta-1)}{\zeta(\delta \beta)}
$$

diverges. It is clear from (1.2) that there is a pole singularity in the sum when $(\delta \beta-1) \rightarrow 1$ from above, that is, when $\beta \rightarrow 2 / \delta$. We conclude, therefore, that the Hausdorff dimension is given by $\beta_{H}=2 / \delta$.

Halsey et al. [6] introduced a method for extracting a spectrum of scalings from experimentally or numerically generated strange (fractal) sets, using a "thermodynamic formalism". They found that the scaling properties of normalized distributions lying upon such sets could be characterized by two indices: $\alpha$, which determines the strength of the singularities, and $f$, which describes how densely they are distributed. The spectrum of singularities is described by giving the range of $\alpha$ values and the function $f=f(\alpha)$.

Shortly thereafter, Feigenbaum [4] pointed out that the work of Halsey et al. was, in fact, the microcanonical version of a thermodynamical formalism that had been introduced by Vul et al. [13] a few years earlier; see also Ruelle [12]. Feigenbaum elucidated the canonical version (canonical paradigm, or $\mathrm{CP}$ for short) in contrast to the microcanonical version (microcanonical paradigm, or MP for short). In this formulation, the probability measure is assumed to be constant on the set. This 
formalism introduces a free energy $F(\beta)$, which is related to $f(\alpha)$ as indicated below. In what follows, we use the notation of Feigenbaum [4].

Consider a dynamical system whose attractor can be hierarchically represented as a set of $N_{n}$ intervals $I_{i}^{(n)}\left(i=1, \ldots, N_{n}\right)$ of lengths $\Delta_{i}^{(n)}$ at the $n$th level. The free energy is defined by

$$
N_{n}{ }^{-F(\beta)}=\sum_{i}\left|\Delta_{i}^{(n)}\right|^{\beta}
$$

where, asymptotically, $F$ becomes independent of $n$. The relation between the MP quantities (Halsey et al. [6]) and the CP quantities (Feigenbaum [4]) is given by

$$
\alpha=\frac{1}{F^{\prime}(\beta)}, \quad f=\beta-\frac{F(\beta)}{F^{\prime}(\beta)}, \quad q=-F(\beta), \quad \tau=-\beta, \quad D_{q}=\frac{\beta}{1+F(\beta)} .
$$

In order to proceed further, we first recall that for a given $Q$, the number of irreducible rationals $P / Q$ is $\phi(Q)$. The Farey series of order $Q,\left\{F_{Q}\right\}$, is a set containing the monotonically increasing sequence of all irreducible rationals $P^{\prime} / Q^{\prime}$ (with $P^{\prime}$ and $Q^{\prime}$ coprime) between 0 and 1 whose denominators do not exceed $Q$ [7]. Next, we define a sequence of integers $\left\{Q_{n}\right\}$ such that, asymptotically,

$$
\frac{Q_{n+1}}{Q_{n}} \rightarrow \sqrt{2}
$$

With these $Q_{n}$, we define a sequence of Farey series $\left\{F_{Q_{n-1}}\right\},\left\{F_{Q_{n}}\right\},\left\{F_{Q_{n+1}}\right\}$ and so on. It follows that the number of rationals contained in $\left\{F_{Q_{n+2}}\right\}$ but not in $\left\{F_{Q_{n+1}}\right\}$ is asymptotically twice the number contained in $\left\{F_{Q_{n+1}}\right\}$ but not in $\left\{F_{Q_{n}}\right\}$. We therefore have a simple prescription for grouping the rationals into distinct exclusive sets $\left\{\left\{F_{Q_{n+1}}\right\}-\left\{F_{Q_{n}}\right\}\right\}$ whose membership increases exponentially fast. We may reexpress this statement by saying that the ratio of the number of rationals contained in each of these consecutive sets is given by

$$
\frac{\sum_{Q_{n+1}}^{Q_{n+2}} \phi(Q)}{\sum_{Q_{n}}^{Q_{n+1}} \phi(Q)} \sim \frac{\int_{Q_{n+1}}^{Q_{n+2}} Q d Q}{\int_{Q_{n}}^{Q_{n+1}} Q d Q}=\frac{Q_{n+2}^{2}-Q_{n+1}^{2}}{Q_{n+1}^{2}-Q_{n}^{2}}=2
$$

asymptotically. We have used $\sum_{Q} \phi(Q) \sim\left(3 / \pi^{2}\right) Q^{2}$ (see [7]) in the large- $Q$ limit.

This construction defines a partition of the rationals from which one may define the free energy $F(\beta)$ in this simple model; asymptotically, this is done through

$$
\begin{aligned}
2^{-F(\beta)} & =\frac{\sum_{Q_{n+1}}^{Q_{n+2}} \phi(Q) Q^{-\delta \beta}}{\sum_{Q_{n}}^{Q_{n+1}} \phi(Q) Q^{-\delta \beta}} \\
& \sim \frac{\int_{Q_{n+1}}^{Q_{n+2}} Q Q^{-\delta \beta} d Q}{\int_{Q_{n}}^{Q_{n+1}} Q Q^{-\delta \beta} d Q}=\frac{\left[Q^{2-\delta \beta}\right]_{Q_{n+1}}^{Q_{n+2}}}{\left[Q^{2-\delta \beta}\right]_{Q_{n}}^{Q_{n+1}}}=\frac{Q_{n+2}^{2-\delta \beta}-Q_{n+1}^{2-\delta \beta}}{Q_{n+1}^{2-\delta \beta}-Q_{n}^{2-\delta \beta}}=(\sqrt{2})^{2-\delta \beta},
\end{aligned}
$$


from which we conclude that

$$
F(\beta)=\frac{\delta \beta}{2}-1
$$

The free energy has the same form as that for a single-scale Cantor set, which is not surprising as there appears to be only a single length scale associated with this model. The Hausdorff dimension is found from the condition $F\left(\beta=\beta_{H}\right)=0$; that is, $\beta_{H}=2 / \delta$, in agreement with the result found from (1.2). Using the $f(\alpha)$ language, we have $\alpha=f(\alpha)=2 / \delta$; that is, the $f(\alpha)$ curve collapses to a single point. It should be noted that the expression for $F(\beta)$ quoted in (1.5) in no way depends on the partition defined through (1.4). Indeed we could choose any $a>1$ such that $Q_{n+1} / Q_{n} \sim \sqrt{a}$, which would lead instead to $a^{-F(\beta)} \sim(\sqrt{a})^{2-\delta \beta}$, and we again have $F(\beta)=\delta \beta / 2-1$.

A natural choice for the $Q_{n}$ that suggests itself is the Fibonacci sequence $Q_{n}=F_{n}$, in which case $Q_{n+1} / Q_{n} \sim 1 / \rho$, where $\rho=(\sqrt{5}-1) / 2$ is the golden mean and $a=1 / \rho^{2}$.

In fact, such a choice has already been used (see [1]) to evaluate the free energy (or its equivalent) and the Hausdorff dimension in the case of the real mode-locking critical circle map; this is a different partition of the rationals which also utilized Farey series. However, the crucial point in both examples is that one may generate partitions of the rationals whose membership increases exponentially fast from one partition to the next. By construction, the variation in size of the denominator $Q$ in the set $\left\{\left\{F_{Q_{n+1}}\right\}-\left\{F_{Q_{n}}\right\}\right\}$ is only of order 1 . In contrast, the Farey level construction which we shall discuss next allows enormous variations in $Q$ at a given level. The smallest denominator is $Q$, while the largest denominator is of order $(1 / \rho) Q$.

The Farey tree level partition of the rationals has frequently been used in discussions of the circle map [1,3], and one can readily calculate the free energy using this scheme. Since the number of rationals doubles from one level to the next, the free energy may be defined through

$$
2^{-F(\beta)}=\frac{\sum_{i}\left[Q_{i}^{(n+1)}\right]^{-\delta \beta}}{\sum_{j}\left[Q_{j}^{(n)}\right]^{-\delta \beta}}
$$

when comparing level $n$ of the Farey tree with level $(n+1)$. The $Q_{j}^{(n)}$ (respectively $Q_{i}^{(n+1)}$ ) are the denominators of the rationals $P_{j}^{(n)} / Q_{j}^{(n)}$ (respectively $P_{i}^{(n+1)} / Q_{i}^{(n+1)}$ ) at level $n$ (respectively $(n+1)$ ). In general, it is not possible to evaluate (1.6) analytically and solve for $F(\beta)$. It is, however, possible to evaluate (1.6) exactly at certain values of $\delta \beta$. From (1.6) it follows that

$$
2^{-F(\beta / \delta)}=\frac{\sum_{i}\left[Q_{i}^{(n+1)}\right]^{-\beta}}{\sum_{j}\left[Q_{j}^{(n)}\right]^{-\beta}} .
$$

This may be evaluated exactly for a number of values of $\beta$. 
(i) $\beta=0$ gives

$$
2^{-F(0)}=\frac{2^{n+1}}{2^{n}}=2,
$$

that is, $F(0)=-1$. Here we have used the fact that the number of rationals at level $n$ in the Farey tree is $2^{n}$. This result for $F(0)$ is in agreement with (1.5) when $\beta$ is set equal to 0 .

(ii) $\beta=-1$ gives

$$
2^{-F(-1 / \delta)}=\frac{\sum_{i} Q_{i}^{(n+1)}}{\sum_{j} Q_{j}^{(n)}}=\frac{3^{n+1}}{3^{n}}=3,
$$

from which we conclude that

$$
F\left(-\frac{1}{\delta}\right)=-\frac{\log 3}{\log 2} .
$$

Here we have used the fact that $\sum_{j} Q_{j}{ }^{(n)} \sim 3^{n}$ (see [1]) at level $n$ of the Farey tree. However, (1.5) predicts that $F(-1 / \delta)=-3 / 2$, which disagrees with (1.8). Therefore, we get different values for the free energy at $\beta=-1$, depending on the partition. It is also possible to evaluate $F(\beta)$ exactly at another point in the Farey tree construction.

(iii) $\beta=-3$ gives

$$
2^{-F(-3 / \delta)}=\frac{\sum_{i}\left[Q_{i}^{(n+1)}\right]^{3}}{\sum_{j}\left[Q_{j}^{(n)}\right]^{3}},
$$

so

$$
F\left(-\frac{3}{\delta}\right)=-\frac{\log 7}{\log 2}
$$

Here we have used the fact that $\sum_{j}\left[Q_{j}{ }^{(n)}\right]^{3} \sim 7^{n}$ (see [1]). The original partition predicts from (1.5) that $F(-3 / \delta)=-5 / 2$, which again disagrees with (1.9). These results are summarized in Table 1 .

TABLE 1 . Thermodynamic free energy $F(\beta)$ evaluated at selected values of $\beta$ for the two partitions with $\delta=2$.

\begin{tabular}{ccc}
\hline$\beta$ & Farey series partition & Farey tree partition \\
\hline 0 & -1 & -1 \\
$-1 / 2$ & $-3 / 2$ & $-\log 3 / \log 2$ \\
$-3 / 2$ & $-5 / 2$ & $-\log 7 / \log 2$ \\
\hline
\end{tabular}

Therefore it is possible to show exactly, by analytical means, that a different partition of the rationals yields different values for the free energy at specific values of $\beta$ (and hence a different singularity spectrum $f(\alpha)$ ) in this simple model for the widths 


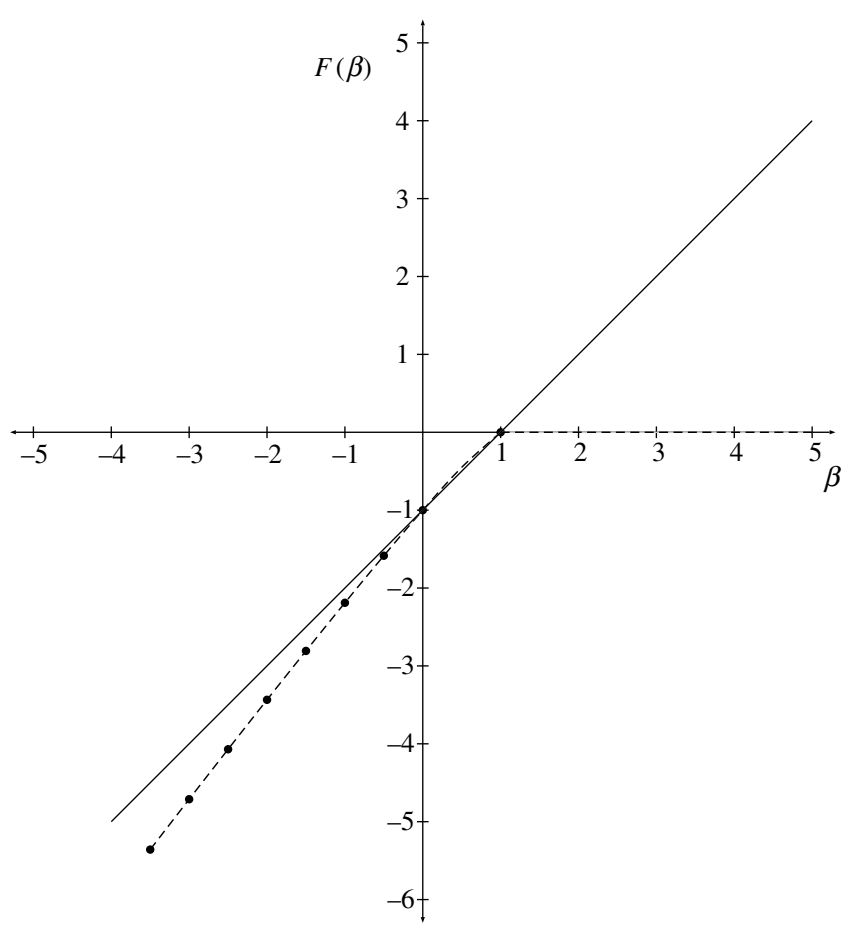

Figure 1. A comparison of the two partitions used to determine $F(\beta)$. The solid line is $F(\beta)=$ $(\beta-1)$, associated with the Farey series/sequence partition. The dashed line shows $F(\beta)$ for the Farey tree partition. This can be calculated analytically for negative half-integers $\beta$ (see Table 1 ) and computationally when $\beta$ is positive for finite-order Farey tree levels; see [1], in which it is conjectured that, asymptotically, $F(\beta)$ converges to $\beta=1$ so that $F(\beta)$ for the Farey tree partition is $C^{1}$ at $\beta=0$. These different partitions lead to different values of thermodynamic free energy $F(\beta)$, with only two points in common: one is at $F(0)=-1$ where all partitions must agree; the other is at $F(1)=0$, which means that both partitions lead to the same Hausdorff dimension $D_{H}=D_{0}=1$. Note that for the purposes of illustration, the specific value $\delta=2$ has been chosen.

of mode-locked intervals. Using (1.3), we can express this in terms of the generalized dimensions $D_{q}$. For the first partition,

$$
D_{q}=\frac{\beta}{1+F(\beta)}=\frac{2}{\delta}
$$

independent of $q$. Equivalently, in terms of MP, (1.5) states that $\tau=(2 / \delta)(q-1)$. Since $\tau=(q-1) D_{q}$ (see [6]), we have $D_{q}=2 / \delta$ once more.

We see that $D_{q}$ is no longer constant. Cvitanović [2] found the $q(\tau)$ (that is, $-F(\beta)$ ) curve for the case $\delta=2$ by using the Farey tree construction together with accelerated convergence numerical techniques. It is clear that in this case $q(\tau)$ is no longer linear in $\tau$, suggesting that there is more than one length scale, which at first sight seems surprising in this simple model. Since $q(\tau)$ is no longer linear in $\tau$ (as it was in the Farey series partition), $D_{q}$ is no longer a constant but varies with $q$. 
TABLE 2. Analytic evaluation of the ratio $\sum_{i}\left[Q_{i}^{(n+1)}\right]^{-2 \beta} / \sum_{j}\left[Q_{j}^{(n)}\right]^{-2 \beta}$ for consecutive levels of the Farey tree in the asymptotic limit $n \rightarrow \infty$ at various values of $\beta$; see [1, Table II].

\begin{tabular}{cc}
\hline$-\beta$ & Asymptotic ratio, or $2^{-F(\beta)}$ \\
\hline 0 & 2 \\
$1 / 2$ & 3 \\
1 & $(5+\sqrt{17}) / 2$ \\
$3 / 2$ & 7 \\
2 & $(11+\sqrt{113}) / 2$ \\
$5 / 2$ & $7+4 \sqrt{6}$ \\
3 & $26.20249 \ldots$ \\
$7 / 2$ & $41.0183 \ldots$ \\
\hline
\end{tabular}

In Figure 1, we plot $q(\tau)$ over a range of $\tau$ values for both partitions of the rationals. The straight line $q=(\tau-1)$ (shown as a dashed line) is associated with the Farey series partition. The other line (continuous) shows $q(\tau)$ computed using Farey tree levels. For the purpose of illustration, the value $\delta=2$ has been chosen. For nonnegative half-integers $\tau, q(\tau)$ is known analytically for the latter partition [1]; see Table 2 . For negative $\tau$, the thermodynamic function is computed for Farey tree levels 13,15 and 17 . Using accelerated convergence techniques, one then obtains $q(\tau)=0$ for $\tau<-1[1,2]$. Clearly, the thermodynamic functions are quite different, and this conclusively demonstrates that their determination depends strongly on the method used to partition the rationals.

In conclusion, we see that by analysing an oversimplified model for the widths of the mode-locked intervals in a critical circle map and partitioning the rationals $P / Q$ in two distinct ways, it is possible to derive two completely different functional forms for $F(\beta)$ (respectively $q(\tau)$ ) which agree at only two distinct values of $\beta$ (respectively $\tau$ ). As noted earlier, one of these values is $\beta=0$ (which was shown analytically in (1.7)) while the other is presumably at $-\tau=\beta=\beta_{H}$. Cvitanović [2] showed that the latter is true for $\delta=2$ to a high precision, by using numerical techniques. Even if one has doubts about such numerical techniques, the ability to compute the thermodynamic function analytically at isolated points and find that the result depends on the form of the partition used demonstrates unequivocally that a serious ambiguity exists. One would expect that partitions other than those discussed in this paper could yield thermodynamic functions that differ from both of the cases depicted in Figure 1.

An alternative way of stating the conclusion is that, when one adopts this simplified model for the mode-locking widths, the Farey series scheme for partitioning the rationals yields a single constant generalized dimension $D_{q}=D_{0}$, while the Farey level tree partition clearly yields a result for $D_{q}$ which is not constant with respect to $q$. Thus, it is clear that there is ambiguity in this attempt to determine the generalized 
dimensions associated with the circle map; the ambiguity presumably persists for the real critical circle map, which is genuinely multifractal.

\section{Acknowledgements}

BGK would like to thank Leo Kadanoff for his hospitality at the James Franck Institute, where part of this work was carried out, as well as for useful discussions and encouragement. He would also like to thank Bob Dewar for the hospitality of the Department of Theoretical Physics at the Australian National University.

\section{References}

[1] R. Artuso, P. Cvitanović and B. G. Kenny, "Phase transitions on strange irrational sets", Phys. Rev. A 39 (1989) 268-281.

[2] P. Cvitanović, Private communication; also see [1].

[3] P. Cvitanović, B. Shraiman and B. Soderberg, "Scaling laws for mode-locking in circle maps", Phys. Scr. 32 (1985) 263-270.

[4] M. J. Feigenbaum, "Some characterizations of strange sets", J. Stat. Phys. 46 (1987) 919-924.

[5] M. J. Feigenbaum, "Scaling spectra and return times of dynamical systems", J. Stat. Phys. 46 (1987) 925-932.

[6] T. C. Halsey, M. H. Jensen, L. P. Kadanoff, I. Procaccia and B. I. Shraiman, "Fractal measures and their singularities: the characterization of strange sets", Phys. Rev. A 34 (1986) 1141-1151.

[7] G. H. Hardy and E. M. Wright, Theory of numbers (Oxford University Press, Oxford, 1938).

[8] H. G. E. Hentschel and I. Procaccia, "The infinite number of generalized dimensions of fractals and strange attractors", Phys. D 8 (1983) 435-444.

[9] M. H. Jensen, P. Bak and T. Bohr, "Complete devil's staircase, fractal dimension, and universality of mode-locking structure in the circle map", Phys. Rev. Lett. 50 (1983) 1637-1639.

[10] M. H. Jensen, P. Bak and T. Bohr, "Transition to chaos by interaction of resonances in dissipative systems. I. Circle maps", Phys. Rev. A 30 (1984) 1960-1969.

[11] O. E. Lanford, "A numerical study of the likelihood of phase-locking", Phys. D 14 (1985) 403-408.

[12] D. Ruelle, Statistical mechanics, thermodynamic formalism (Addison-Wesley, Reading, MA, 1978).

[13] E. B. Vul, Ya. G. Sinai and K. M. Khanin, "Feigenbaum universality and thermodynamic formalism", Uspekhi Mat. Nauk 39 (1984) 3-37.

[14] E. B. Vul, Ya. G. Sinai and K. M. Khanin, Russian Math. Surveys 39 (1984) 1-40. 